\title{
Multi-constellation GNSS orbit combination based on MGEX products
}

\author{
Gustavo Mansur ${ }^{1,2}$, Pierre Sakic ${ }^{1}$, Benjamin Männel ${ }^{1}$, and Harald Schuh ${ }^{1,2}$ \\ ${ }^{1}$ Helmholtz Centre Potsdam - GFZ German Research Centre for Geosciences, \\ Section 1.1 - Space Geodetic Techniques, Potsdam, Germany \\ ${ }^{2}$ Technische Universität Berlin, Institute for Geodesy and Geoinformation Technology, Faculty VI, Berlin, Germany
}

Correspondence: Gustavo Mansur (mansur@gfz-potsdam.de) and Pierre Sakic (pierre.sakic@gfz-potsdam.de)

Received: 29 May 2019 - Revised: 23 December 2019 - Accepted: 24 January 2020 - Published: 14 February 2020

\begin{abstract}
The mission of the International GNSS Service (IGS) is to deliver highly accurate GNSS data and products to the scientific users and the community. Among these products, precise orbits, and clocks for GPS and GLONASS are available to the public. These products are system-wise combinations based on solutions provided by the Analysis Centers (AC). Over the past years, the IGS has been putting efforts in extending the service to other navigation satellite systems within the Multi-GNSS Experiment and Pilot Project (MGEX). Several ACs contribute by providing solutions containing not only GPS and GLONASS but also Galileo, BeiDou, and QZSS. However, there is no official MGEX combination so far. Therefore, we started to develop a new combination algorithm aiming at a fully consistent multi-constellation solution. We apply two different strategies focusing on the alignment of the orbits to the International Terrestrial Reference Frame (ITRF). In the first strategy, we use the Earth Rotation Parameters (ERP) to align the orbits, whereas in the second strategy Helmert parameters provided by the Terrestrial Frame Combination Center (TFCC) are applied. Based on the alignment we compare the GPS orbit products from both strategies with the official IGS orbits. These preliminary results show that the ERP strategy agrees with the official orbits around by $30 \mathrm{~mm}$ whereas, with the second strategy, the agreement is around $15 \mathrm{~mm}$.
\end{abstract}

\section{Introduction}

Over the past decade, new Global Navigation Satellite Systems (GNSS) emerged and became operational in recent years. For example, the European system Galileo that de- clared the initial operability in 2016 (ESA, 2016) and the Chinese BeiDou provide global services since the end of 2018 (CSNO, 2018). Besides, some Regional Navigation Satellite Systems (RNSS) like the Japanese Quasi-Zenith Satellite System (QZSS) and the Indian Regional Navigation Satellite System (NAVIC) are officially available, respectively, since November 2018 (National Space Policy Secretariat, 2019) and April 2016 (Rao Satellite Centre, 2019). The new constellations open additional possibilities, for example, a larger number of satellites covering the sky, new advances in signal structure and frequencies, clocks with better performance and improved determination of geodetic parameters, since different systems have different orbital planes, altitudes and orbital periods (Montenbruck et al., 2017). These are benefits to all kind of applications that requires GNSS products.

Since 1994, highly accurate GNSS products are provided by the International GNSS Service (IGS), a federation of over 200 geodetic and space institutes (Johnston et al., 2017). From the very beginning the IGS provides, orbits products for GPS on an operational basis. Starting with the IGEX'98 experiment, IGS put efforts also to integrate the GLONASS constellation into operational service. The orbits are determined by the ACC (Analysis Center Coordinator) as a combination of several independent solutions submitted by the IGS Analysis Centers (AC), which we will refer to here as legacy combination. Regarding the GLONASS combination, it has to be mentioned that this solution is still experimental and is performed separately from the GPS combination. Since 2012, the IGS has been putting efforts in extending the service to the new navigation satellite systems within the IGS Multi-GNSS Experiment and Pilot Project (MGEX, 
Montenbruck et al., 2017). As part of MGEX, a number of ACs provide solutions containing also BeiDou, Galileo and QZSS constellations. Although some investigations in methods to combine the MGEX products have been carried out (Fritsche, 2016; Sakic et al., 2018), there is no official MGEX combination so far.

In this article, we describe preliminary studies for a new algorithm to achieve a combined orbit and clock product within MGEX, that we will call "ECG" combination (for Experimental Combination GFZ). We perform investigations on how to align the orbits properly concerning the International Terrestrial Reference Frame (ITRF, Altamimi et al., 2016). This alignment is a pre-requisite to make the combined solution comparable to the results offered by the IGS in their legacy products. For this study orbits from GPS week 1850 (end of June 2015) until week 1960 (beginning of August 2017) are combined. First using Earth Rotation Parameters (ERP) and second using rotation parameters coming from station coordinates (Helmert transformation). Based on the alignment, the combination is performed.

The paper is organized as follows: Section 2 presents a small review of the distribution of the MGEX products and a comparison between the legacy and MGEX provided by the ACs. Section 3 proposes two strategies to align the ACs' orbits and then perform the combination. The combinations are assessed based on the RMS between the individual solution and the combined orbit. Section 4 is dedicated to our conclusions and perspectives.

\section{ACs' MGEX products}

So far, six ACs provide multi-constellation products to the IGS/MGEX, namely:

- Deutsches GeoForschungsZentrum (GFZ), Potsdam (Uhlemann et al., 2015; Deng et al., 2016)

- Centre National d'Etudes Spatiales (CNES), Groupe de Recherche de Géodésie Spatiale (GRGS), Toulouse (Loyer et al., 2018; Katsigianni et al., 2018);

- Center for Orbit Determination in Europe (CODE), Bern (Prange et al., 2015, 2018);

- Technische Universität München (TUM), Munich (Selmke et al., 2018);

- Shanghai Astronomical Observatory (SHAO), Shanghai (Chen et al., 2012);

- Wuhan University (WU), Wuhan (Guo et al., 2016).

Table 1 summarizes the status and characteristics of the delivered products. Among these, the satellite positions in the Earth-fixed (Cartesian) frame with 15 or 5 min rate are provided in . $\mathrm{sp} 3$ format and clock corrections (. $\mathrm{clk}$ format) with $30 \mathrm{~s}$ sampling rate. Earth rotation parameters, in particular the daily estimates for $X$ and $Y$ pole offsets and rates are given in . erp format while station coordinates are provided in a SINEX format $(. \operatorname{sn} x)$. Inter-systems biases describing time reference differences between observations of different GNSS are provided in .bias format. Three of the six ACs - GRGS, GFZ, CODE - also provide GPS and GLONASS products to the legacy IGS processing line. For each of those three ACs, we extract the GPS orbit information from the final legacy products as well as the GPS orbits from their MGEX files and compute the RMS 3-D between both solutions (Fig. 1). As GRGS extracts their IGS legacy submission from their MGEX products their solutions are identical and therefore not shown in Fig. 1. For CODE and GFZ, the differences are at the centimeter level. For the CODE differences a significant decrease over time is visible, what we may attribute to the changes and improvements in the determination of their MGEX orbits, for example, the usage of a new model for solar radiation pressure as described in Arnold et al. (2015) and Prange et al. (2017).

\section{Orbits alignment}

Since the IGS started to distribute orbit products, they are provided in the International Terrestrial Reference Frame (ITRF). Over the years two different strategies were applied to align all the ACs products and consequently the final combined orbits to the ITRF (Kouba et al., 1994; Kouba, 2015). To keep the equivalence, the combined MGEX orbits should be in the ITRF. To carry through this, we study in this section the two alignment methods and we perform the orbit combination.

\subsection{ERP based alignment}

At the very beginning of the IGS orbits combination, when ACs did not submit the estimated station coordinates the ERP strategy was developed to align orbits to the ITRF. The method is discussed and described in Springer and Beutler (1993), Kouba et al. (1994) and Beutler et al. (1995). Based on the Bulletin B, provided by the International Earth Rotation and Reference Systems Service (IERS) (Luzum and Gambis, 2014), and the ERP solutions provided by the ACs, each orbit is aligned to the ITRF. This pole rotation is described in Xu (2007). For further comparisons, we ignored TUM and SHAO as they do not provide ERP which is a prerequisite for this first combination strategy. The combination itself is performed with the following steps (Fig. 2). In a first step, a mean orbit of all ACs solutions is computed for each satellite. To remove small systematic errors a Helmert transformation is performed between the individual AC orbits and the derived mean position. Based on this transformation the weights of the ACs is computed using the following equation 
Table 1. Analysis Centers and their product submissions, as reference also IGS legacy and MGX (Sakic et al., 2018) are added.

\begin{tabular}{|c|c|c|c|c|c|c|c|}
\hline AC/Prod. & AC abbreviation & Orbits & ERP & Clock corrections & SINEX & Bias & Constellations \\
\hline CODE & COD & $\checkmark$ & $\checkmark$ & $\checkmark$ & & $\checkmark$ & $\mathrm{G}+\mathrm{R}+\mathrm{E}+\mathrm{C}+\mathrm{J}$ \\
\hline GFZ & GFZ & $\checkmark$ & $\checkmark$ & $\checkmark$ & & $\checkmark$ & $\mathrm{G}+\mathrm{R}+\mathrm{E}+\mathrm{C}+\mathrm{J}$ \\
\hline GRGS & GRG & $\checkmark$ & & $\checkmark$ & $\checkmark$ & & $\mathrm{G}+\mathrm{R}+\mathrm{E}$ \\
\hline SHAO & SHA & $\checkmark$ & & $\checkmark$ & & & $\mathrm{G}+\mathrm{R}+\mathrm{E}+\mathrm{C}$ \\
\hline TUM & TUM & $\checkmark$ & & $\checkmark$ & & & $\mathrm{E}+\mathrm{C}+\mathrm{J}$ \\
\hline WU & WUM & $\checkmark$ & $\checkmark$ & $\checkmark$ & & & $\mathrm{G}+\mathrm{R}+\mathrm{E}+\mathrm{C}+\mathrm{J}$ \\
\hline IGS legacy & IGS & $\checkmark$ & $\checkmark$ & $\checkmark$ & $\checkmark$ & & $\mathrm{G}+(\mathrm{R})$ \\
\hline MGX (Sakic et al., 2018) & MGX & $\checkmark$ & & & & & $\mathrm{G}+\mathrm{R}+\mathrm{E}+\mathrm{C}+\mathrm{J}$ \\
\hline
\end{tabular}

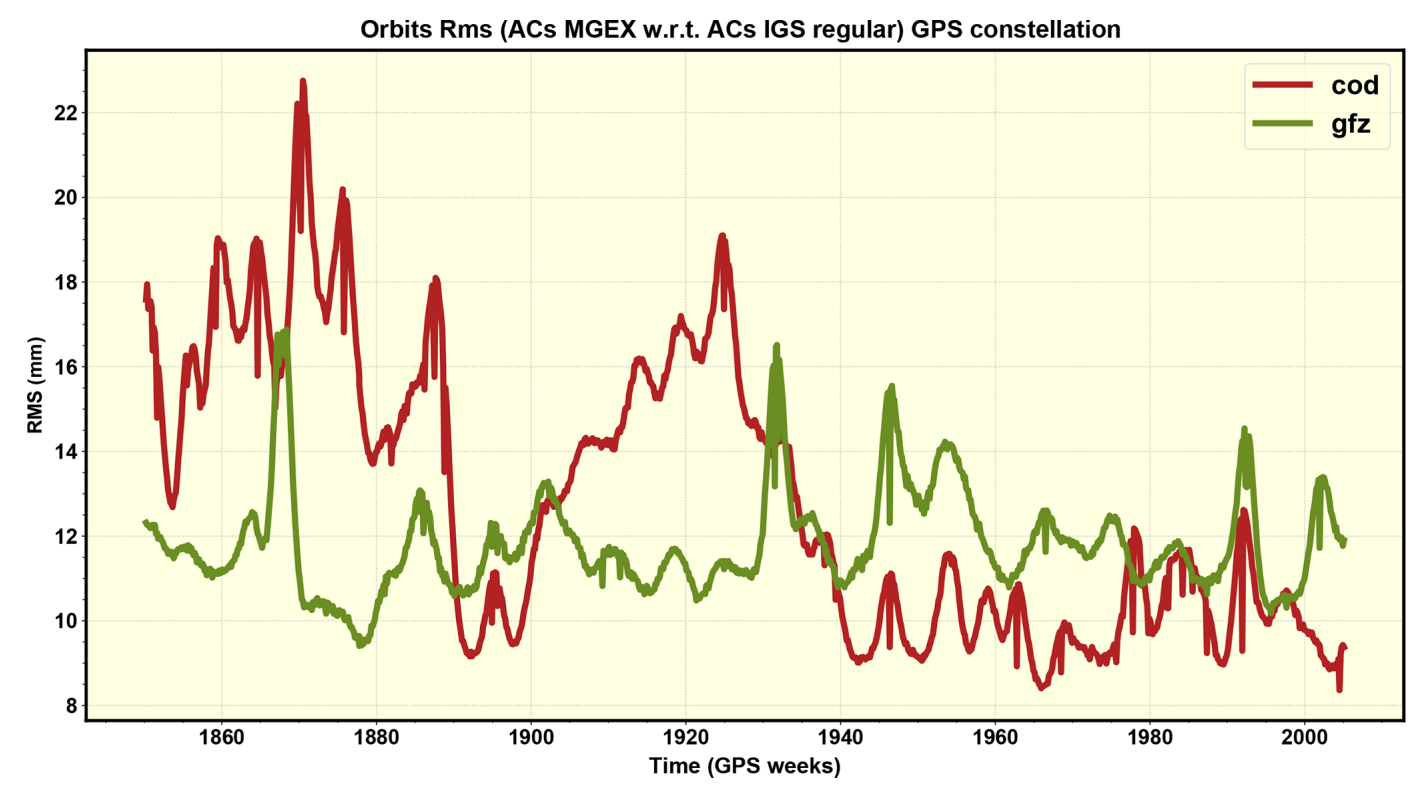

Figure 1. Difference between the orbits from the IGS legacy products and the MGEX products, submitted by two ACs (CODE and GFZ) GPS constellation.

from Kouba et al. (1994):

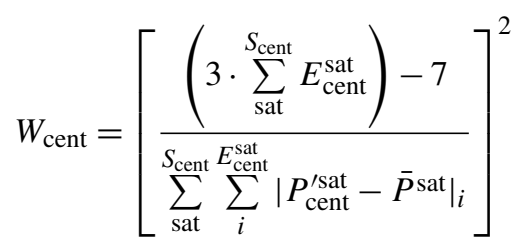

In Eq. (1) $S_{\text {cent }}$ is the number of satellites per $\mathrm{AC}, E_{\text {cent }}^{\mathrm{sat}}$ is the number of positions per AC per satellite. The position of the transformed orbits and the mean orbit is represented by $P_{\text {cent }}^{\text {sat }}$ and $\bar{P}^{\text {sat }}$, respectively. Therefore, the weighting relies on the geometrical differences between AC solutions and mean orbits. With the ACs' weights, the weighted mean orbits are calculated. The two final steps are to compute the transformations between the weighted mean orbits and the individual orbits and then calculate a final weighted average orbit. More details about the specific method are provided in Kouba et al. (1994).
Figure 3 represents the RMS of orbit differences between individual ACs solutions and the final combined orbits. For the comparison, we added two additional solutions to this figure. The first solution is the legacy IGS (GPS only) product, the second solution is the MGEX combination based on Sakic et al. (2018), called MGX. For most of the ACs, a constant behavior with RMS values around $15 \mathrm{~mm}$ is visible. Considering the legacy and MGX solutions, the RMS is around $25 \mathrm{~mm}$. We assigned this higher value compared with the ACs solutions to the different set of orbits used in the processing chain. The first one is based on the rotations coming from the parameters of a Helmert transformation, which will be discussed in more detail in the next section, and the second one is based on the ERP combination provided by the IGN (Rebischung et al., 2016).

Figure 4 shows that the RMS of the ACs' orbits are decreasing over time and that they are getting closer to the ECG solution indicating that the orbit consistency between the ACs improved. Among all the constellations, the GPS orbits 


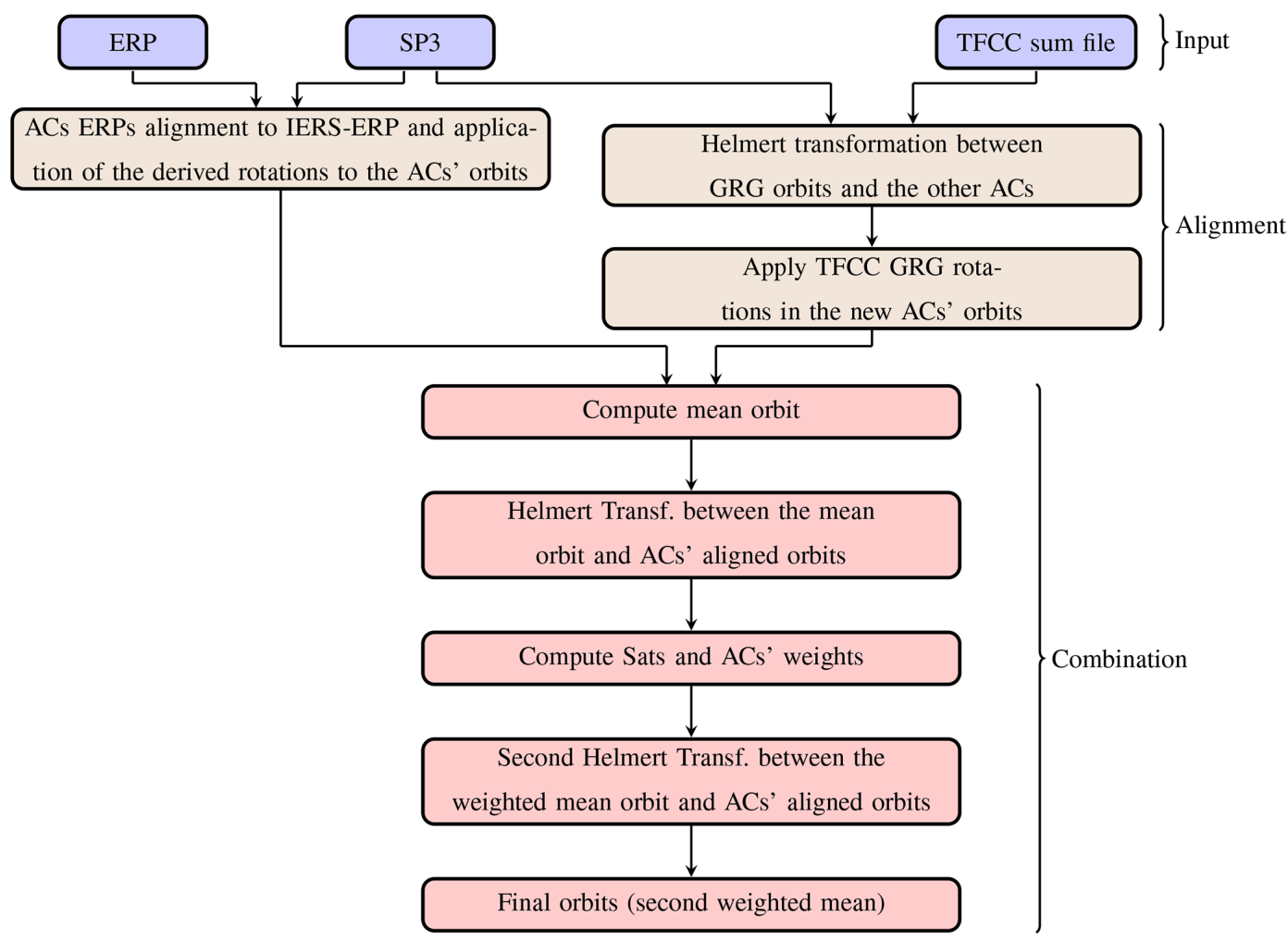

Figure 2. The flowchart presents the generalized steps for both alignment strategies and orbit combination.

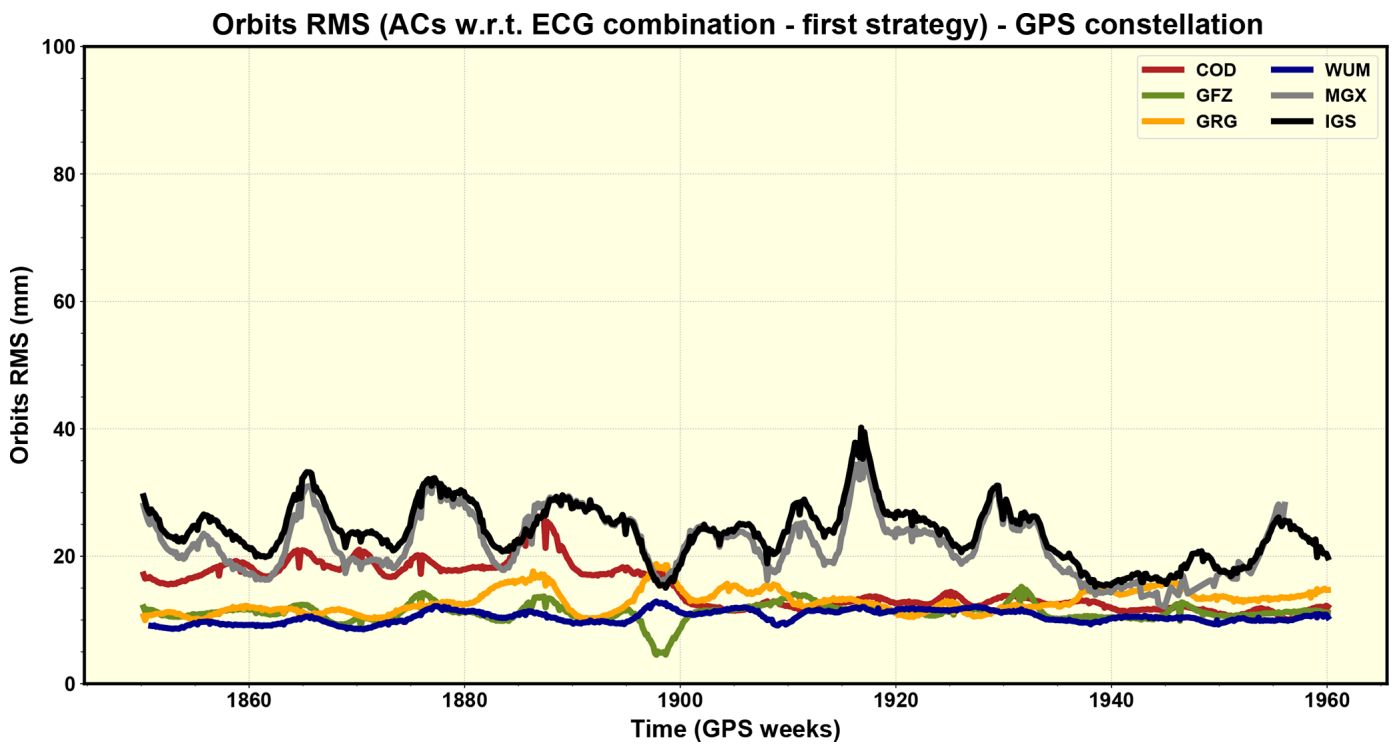

Figure 3. RMS values with respect to the individual ACs' MGEX submission for GPS orbits and the GPS ECG combination result computed by ERP strategy.

seem to be the most stable followed by Galileo, GLONASS, and BeiDou. However, the orbit quality varies from week to week and so does the agreement to the combined solution. This issue might be related to inaccurate models decreasing the orbit quality, such as the handling of solar radiation pres- sure where inaccuracies might result in stronger orbit errors during periods of low elevation of the Sun above the satellite orbit plane. Some investigations have shown for instance that a mismodelling for Galileo satellites during those periods can also affect the orbit determination of other constella- 


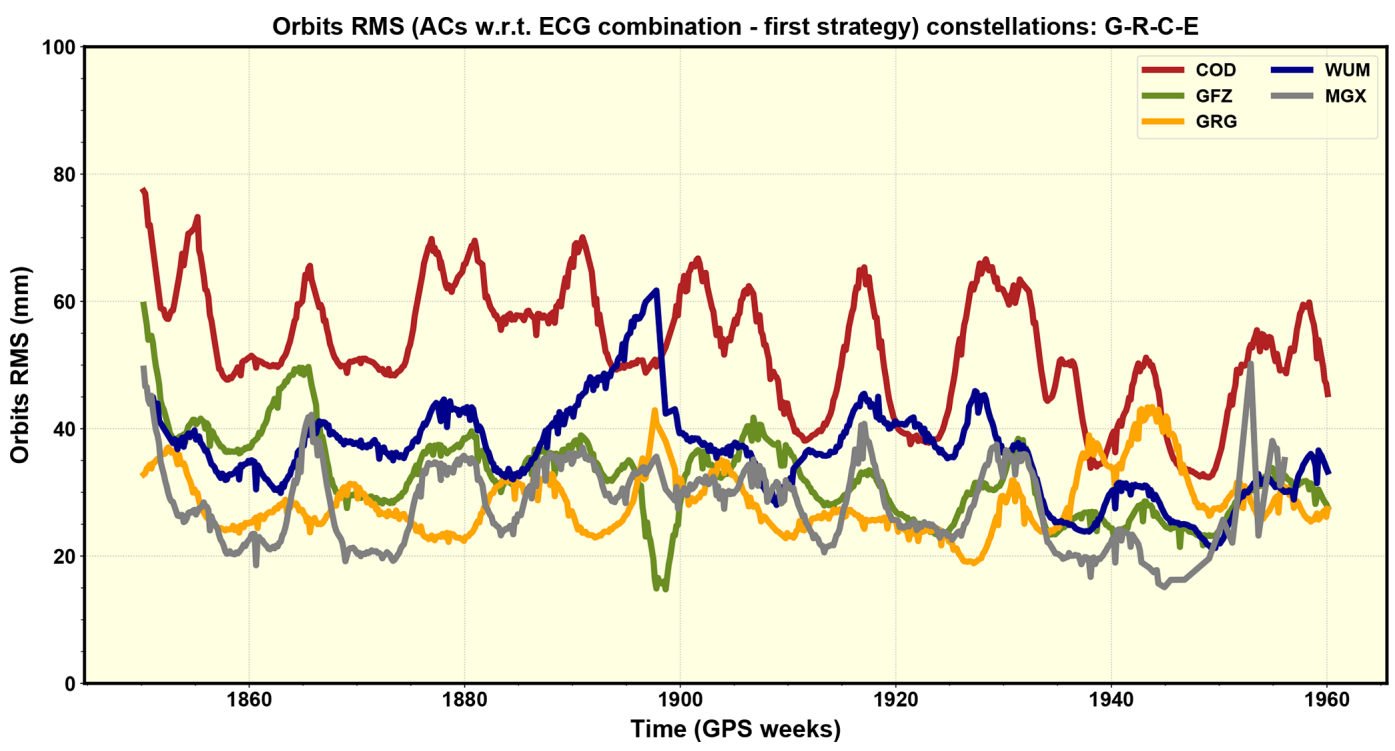

Figure 4. RMS values with respect to the individual ACs' MGEX submission orbits using all constellations and the ECG combination results computed by the ERP strategy.

tions (Sidorov et al., 2018, 2019). Regarding that or problems in the attitude model resulting in orbit discrepancies during eclipse periods. Over the weeks of the study, the weight distribution is similar among the four ACs, where GRGS received the highest value of around $31 \%$.

\subsection{Helmert transformation parameter based alignment}

This strategy relies on the alignment method currently used by the IGS for the final products, where nowadays are provided in the IGS reference frame which is consistent with the ITRF. In the combination process, each AC is pre-aligned according to three rotations coming from a set of Helmert parameters. These rotations are based on the station coordinates that the ACs provide in daily SINEX files. The TFCC (Terrestrial Frame Combination Center, https://webigs.ign.fr/ tfcc/, last access: 13 February 2020), operated by the IGN, performs the alignment of the station coordinates and weekly distributes the transformation parameters in the summary file. In the MGEX contribution the SINEX files are not available for most of the ACs Table (Table 1). However, as GRGS provides SINEX files and the corresponding IGS and MGEX submissions are identical, we developed a workaround approach. As shown in Fig. 2, the first step of the alignment consists of a seven parameter Helmert transformation (Molodensky et al., 1962) between the orbits of each AC and those of GRGS. With all the orbits aligned to the GRGS, we applied the TFCC rotations. The orbit combination was then performed as described in the ERP strategy section.

Comparing both strategies in general, the differences are small when regarding the RMS values to each orbit. For the GPS constellation shown in Fig. 5, we see smaller RMS in the second alignment than in ERP alignment for the comparison to legacy $(\mathrm{G})$ and $M G X$ orbits. The RMS values are around $15 \mathrm{~mm}$ while reaching $25 \mathrm{~mm}$ in the first approach. This result indicates that rotating the orbits to the GRGS products is a good approximation of the official ITRF alignment and still relies on an official rotation. In Fig. 6, the ACs' orbits for all constellations $(\mathrm{G}+\mathrm{R}+\mathrm{E}+\mathrm{C})$ show RMS values very similar compared to Fig. 4 of around $50 \mathrm{~mm}$ and few millimeters higher for CODE.

\section{Conclusions and further investigations}

We are developing a new orbit and clock combination software which allows deriving a consistent orbit solution for all GNSS constellations at the same time. Hence we study two ways to extend the combination methodology. In a first test, we combined MGEX orbits from week 1850-1960 for GPS, GLONASS, Galileo, and BeiDou. The alignment of the MGEX products to the ITRF is a critical topic that needs to be studied in a way to find the best solution in terms of available products and consistency. Having products in the official reference system is, of course, essential to any application in geodesy, surveying, positioning, etc. Both ERP strategy and Helmert-based strategy worked well, while we found a better agreement to the IGS legacy when applying the second strategy, it is an expected result since the second strategy aims to reproduce a similar workflow as the one used for the IGS legacy final orbits. Comparing the derived combined orbits shows an overall agreement at the level of $15 \mathrm{~mm}$ to the IGS legacy (GPS only) and of $50 \mathrm{~mm}$ between the different MGEX solutions (all constellations). It seems that the actual differences between legacy and the MGEX products 


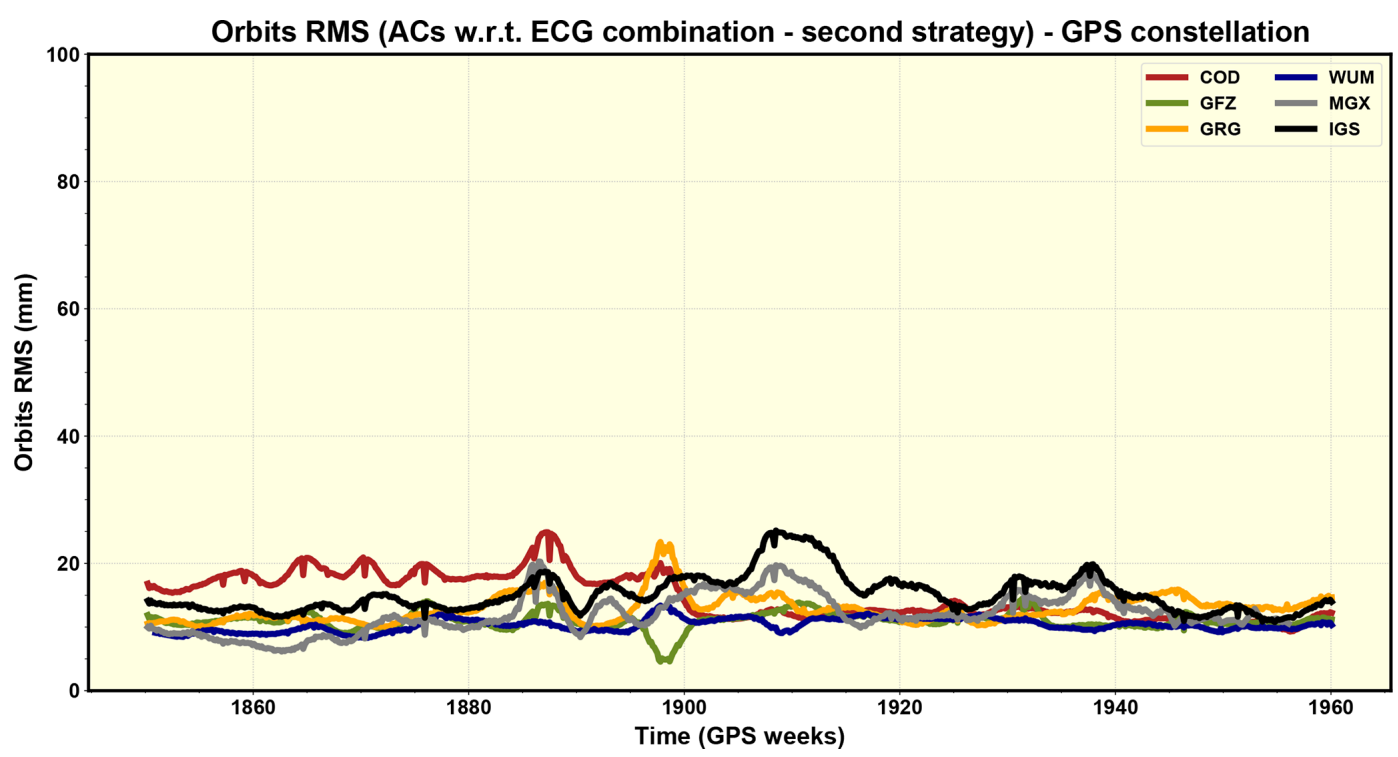

Figure 5. RMS with respect to the individual ACs' MGEX submission GPS orbits and the GPS ECG combination results computed by the Helmert-based strategy.

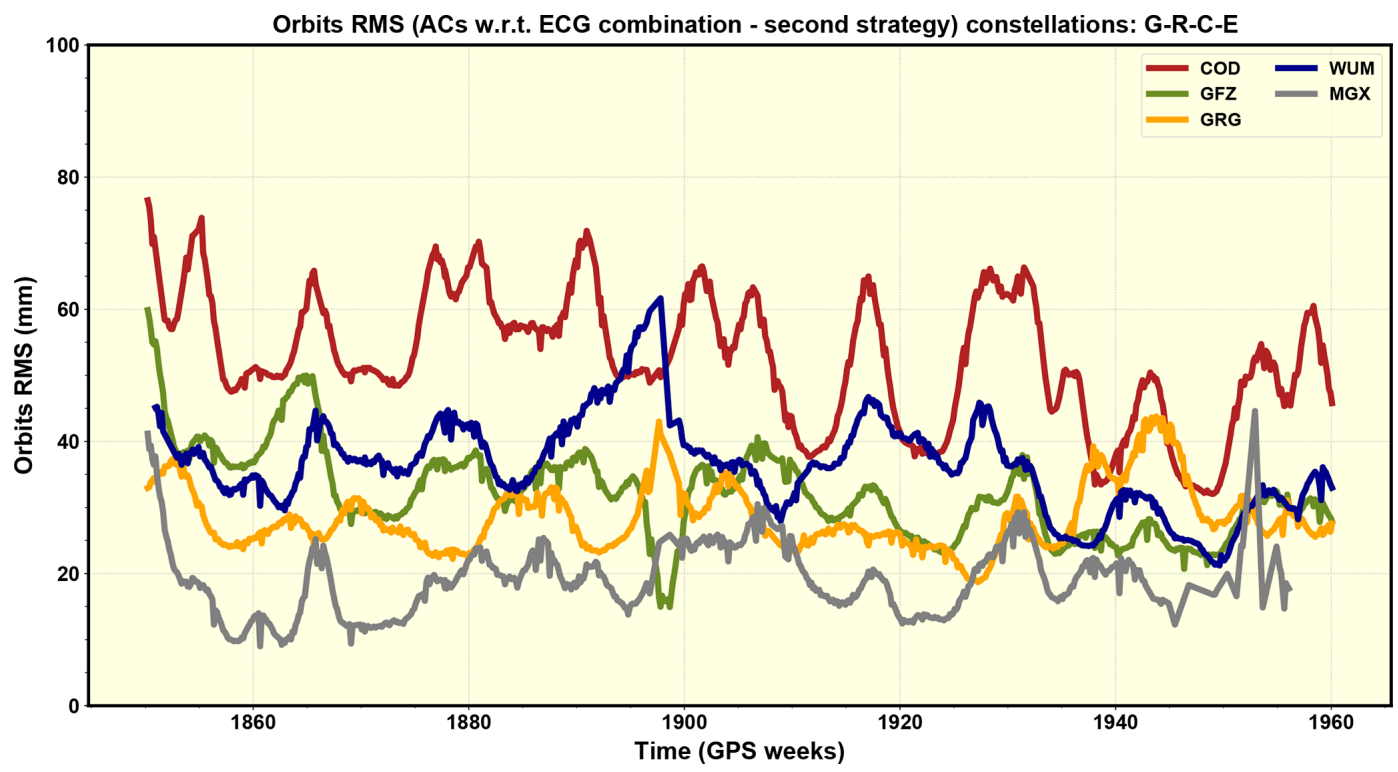

Figure 6. RMS with respect to the individual ACs' orbits using all constellations and the ECG combination result using the Helmert-based strategy.

are around $10 \mathrm{~mm}$. Because of that, we could not expect our combination to reach a millimeter level difference when we compare with the GPS legacy orbits. Moreover, our results showed that it is possible to have a similar methodology as the one existing currently to the GPS/GLONASS official orbits, where the alignment could be done using the rotations coming from a Helmert transformation if all the ACs would provide SINEX files. As expected, the orbits provided by the ACs are most stable for GPS satellites. For the other systems, we noticed that Galileo has the strongest improvements over time, where the ACs RMS values decreased from $80 \mathrm{~mm}$ in the week 1860 to around $40 \mathrm{~mm}$ in the week 1960.

Regarding the implementation of the algorithm to combine the MGEX products, we are currently studying a suitable weighting scheme taking into account the characteristics of each system. Weights will be computed depending not only on the ACs and satellites but also on the constellations. Furthermore, we will test independent weighting strategies for the final orbits, for example, based on residuals derived in an orbit validation using satellite laser ranging observations 
(possible for GLONASS, Galileo, and BeiDou). To achieve the highest consistency our goal is to include also the clock and bias products in the combination process.

Data availability. The data used for this work are publicly and freely available on the MGEX section of the IGS data centers (e.g. ftp://cddis.gsfc.nasa.gov/pub/gps/products/mgex/, last access: 13 February 2020) (Montenbruck et al., 2017). The combined products described in this study can be provided for free on demand.

Author contributions. GM, PS and BM designed the study. GM and PS implemented the algorithm. GM wrote the paper. All the authors joined research discussion and gave their feedback for the paper writing.

Competing interests. The authors declare that they have no conflict of interest.

Special issue statement. This article is part of the special issue "European Geosciences Union General Assembly 2019, EGU Geodesy Division Sessions G1.1, G2.4, G2.6, G3.1, G4.4, and G5.2". It is a result of the EGU General Assembly 2019, Vienna, Austria, 712 April 2019.

Acknowledgements. We would like to thank the International GNSS Service and more particularly the MGEX Analysis Centers for providing their products. The PhD of Gustavo Mansur is funded by the Deutscher Akademischer Austauschdienst (DAAD, German Academic Exchange Service).

Financial support. The article processing charges for this openaccess publication were covered by a Research Centre of the Helmholtz Association.

Review statement. This paper was edited by Adrian Jaeggi and reviewed by Tim Springer and one anonymous referee.

\section{References}

Altamimi, Z., Rebischung, P., Métivier, L., and Collilieux, X.: ITRF2014: A new release of the International Terrestrial Reference Frame modeling nonlinear station motions, J. Geophys. Res.-Sol. Ea., 121, 6109-6131, https://doi.org/10.1002/2016JB013098, 2016.

Arnold, D., Meindl, M., Beutler, G., Dach, R., Schaer, S., Lutz, S., Prange, L., Sośnica, K., Mervart, L., and Jäggi, A.: CODE's new solar radiation pressure model for GNSS orbit determination, J. Geodesy, 89, 775-791, https://doi.org/10.1007/s00190015-0814-4, 2015.
Beutler, G., Kouba, J., and Springer, T.: Combining the orbits of the IGS Analysis Centers, B. Geod., 69, 200-222, https://doi.org/10.1007/BF00806733, 1995.

Chen, J., Wu, B., Hu, X., and Li, H.: SHA: The GNSS Analysis Center at SHAO, in: China Satellite Navigation Conference (CSNC) 2012 Proceedings, edited by: Sun, J., Jiao, W., $\mathrm{Wu}, \mathrm{H}$. , and Shi, C.,Lecture Notes in Electrical Engineering, Springer Berlin Heidelberg, Berlin, Heidelberg, 243, 213-221, https://doi.org/10.1007/978-3-642-29175-3_19, 2012.

CSNO: The BDS-3 Preliminary System Is Completed to Provide Global Services, available at: http://en.beidou.gov. cn/WHATSNEWS/201812/t20181227_16837.html (last access: 13 February 2020), 2018.

Deng, Z., Fritsche, M., Nischan, T., and Bradke, M.: Multi-GNSS ultra rapid orbit-, clock-\& EOP-product series, GFZ Data Services, Potsdam, available at: http://dataservices.gfz-potsdam.de/ panmetaworks/showshort.php?id=escidoc: 1739888 (last access: 13 February 2020), https://doi.org/10.5880/GFZ.1.1.2016.003, 2016.

ESA: Galileo begin serving the globe, available at: http://www.esa.int/Our_Activities/Navigation/Galileo_begins_ serving_the_globe (last access: 13 February 2020), 2016.

Fritsche, M.: Multi-GNSS orbit and clock combination: Preliminary results, in: IGS Workshop 2016, Sydney, Australia, 2016.

Guo, J., Xu, X., Zhao, Q., and Liu, J.: Precise orbit determination for quad-constellation satellites at Wuhan University: strategy, result validation, and comparison, J. Geodesy, https://doi.org/10.1007/s00190-015-0862-9, 2016.

Johnston, G., Riddell, A., and Hausler, G.: The International GNSS Service, in: Springer Handbook of Global Navigation Satellite Systems, pp. 967-982, Springer International Publishing, Cham, https://doi.org/10.1007/978-3-319-42928-1_33, 2017.

Katsigianni, G., Loyer, S., Perosanz, F., Mercier, F., Zajdel, R., and Sośnica, K.: Improving Galileo orbit determination using zero-difference ambiguity fixing in a Multi-GNSS processing, Adv. Space Res., 63, 2952-2963, https://doi.org/10.1016/j.asr.2018.08.035, 2018.

Kouba, J.: A Guide to using international GNSS Service (IGS) Products, Geodetic Survey Division Natural Resources, Canada, Ottawa, 6, 34 pp., 2015.

Kouba, J., Mireault, Y., and Lahaye, F.: IGS orbit/clock combination and evaluation, Appendix 1 of the analysis coordinator report, international GPS Service For Geodynamics 1994 Annual Report, Jet Propulsion Laboratory publication, pp. 18-95, 1994.

Loyer, S., Perosanz, F., Versini, L., Katsigianni, G., Mercier, F., and Mezerette, A.: CNES/CLS IGS Analysis center: recent activities, in: IGS Workshop 2018, Wuhan, 2018.

Luzum, B. and Gambis, D.: Explanatory Supplement to Bulletins A and Bulletin B/C04, Tech. rep., Observatoire de Paris, Paris, 2014.

Molodensky, M., Eremeev, V., and Yurkina, M.: Methods for the Study of the External Gravitational Field and Figure of the Earth, Israel Program for Scientific Translations, Jerusalem, 1962.

Montenbruck, O., Steigenberger, P., Prange, L., Deng, Z., Zhao, Q., Perosanz, F., Romero, I., Noll, C., Stürze, A., Weber, G., Schmid, R., MacLeod, K., and Schaer, S.: The Multi-GNSS Experiment (MGEX) of the International GNSS Service (IGS) - Achievements, prospects and challenges, Adv. Space Res., 59, 1671-1697, https://doi.org/10.1016/j.asr.2017.01.011, 2017 
(data available at: ftp://cddis.gsfc.nasa.gov/pub/gps/products/ mgex/, last access: 13 February 2020).

National Space Policy Secretariat, C. O.: Start of QZSS Services, available at: https://sys.qzss.go.jp/dod/en/constellation.html (last access: 13 February 2020), 2019.

Prange, L., Dach, R., Lutz, S., Schaer, S., and Jäggi, A.: The CODE MGEX Orbit and Clock Solution, : IAG 150 Years. International Association of Geodesy Symposia, Springer, Cham., 143, 767773, https://doi.org/10.1007/1345_2015_161, 2015.

Prange, L., Orliac, E., Dach, R., Arnold, D., Beutler, G., Schaer, S., and Jäggi, A.: CODE's five-system orbit and clock solution - the challenges of multi-GNSS data analysis, J. Geodesy, 91, 345-360, https://doi.org/10.1007/s00190-016-0968-8, 2017.

Prange, L., Arnold, D., Dach, R., Schaer, S., Sidorov, D., Stebler, P., Villiger, A., and Jäggi, A.: CODE product series for the IGSMGEX project, Astronomical Institute, University of Bern, Bern, https://doi.org/10.7892/boris.75882.2, 2018.

Rao Satellite Centre, D. O. S.: IRNSS - Indian Regional Navigation Satellite System, available at: https://www.isac.gov.in/ navigation/irnss.jsp (last access: 13 February 2020), 2019.

Rebischung, P., Altamimi, Z., Ray, J., and Garayt, B.: The IGS contribution to ITRF2014, J. Geodesy, 90, 611-630, https://doi.org/10.1007/s00190-016-0897-6, 2016.

Sakic, P., Mansur, G., Viegas, E., Männel, B., and Schuh, H.: Towards a Multi-Constellation combination: Improving the IGS orbit and clock combination software for MGEX products, in: IGS Workshop 2018, Wuhan, https://doi.org/10.13140/RG.2.2.10510.41284/1, 2018.
Selmke, I., Duan, B., and Hugentobler, U.: Status of the TUM MGEX orbit and clock products, in: IGS Workshop 2018, Wuhan, https://doi.org/10.1007/s00190-014-0774-0, 2018.

Sidorov, D., Dach, R., Prange, L., and Jäggi, A.: Improved orbit modelling of Galileo satellites during eclipse seasons, IGS Workshop 2018, https://doi.org/10.7892/boris.121104, 2018.

Sidorov, D., Dach, R., Prange, L., and Jäggi, A.: Advancing the orbit model for Galileo satellites during the eclipse seasons, in: Swiss National Report on the Geodetic Activities in the years 20152019, edited by: Mueller-Gantenbein, J., Brockmann, E., Marti, U., Rothacher, M., and Merminod, B., pp. 38-39, Swiss Geodetic Commission, XXVII General Assembly of the IUGG, Montreal, 2019.

Springer, T. A. and Beutler, G.: Towards an official IGS orbit by combining the results of all IGS Processing Centers, in: Proceedings of the 1993 IGS Workshop, March 1993, pp. 24-26, 1993.

Uhlemann, M., Gendt, G., Ramatschi, M., and Deng, Z.: GFZ Global Multi-GNSS Network and Data Processing Results, in: International Association of Geodesy Symposia, 12, 673-679, https://doi.org/10.1007/1345_2015_120, 2015.

$\mathrm{Xu}$, G.: GPS, Springer Berlin Heidelberg, Berlin, Heidelberg, https://doi.org/10.1007/978-3-540-72715-6, 2007. 